\title{
Dual-hop Relaying Networks for Short-Packet URLLCs: Performance analysis and Optimization
}

\author{
Nguyen Thi Yen Linh, Ngo Hoang Tu, Pham Ngoc Son, and Vo Nguyen Quoc Bao, Senior Member, IEEE
}

\begin{abstract}
This paper investigates short-packet communications for the dual-hop decode-and-forward relaying system to facilitate ultra-reliable and low-latency communications. In this system, a selected relay having the highest signal-to-noise ratio (SNR) serves as a forwarder to support the unavailable direct link between the source and destination, whereas a maximum ratio combining technique is leveraged at the destination to achieve the highest diversity gain. Approximated expressions of end-to-end (e2e) block error rates (BLERs) are derived over quasi-static Rayleigh fading channels and the finite-blocklength regime. To gain more insights about the performance behavior in the high-SNR regime, we provide the asymptotic analysis with two approaches, from which the qualitative conclusion based on the diversity order is made. Furthermore, the power allocation and relay location optimization problems are also considered to minimize the asymptotic e2e BLER under the configuration constraints. Our analysis is verified through Monte-Carlo simulations, which yield the system parameters' impact on the system performance.
\end{abstract}

Index Terms-UItra-reliable and low-latency, short-packet communications, relay network, optimization.

\section{INTRODUCTION}

$\mathbf{I}$ $\mathrm{N}$ the dramatical development of emerging communication systems, the technologies that support the fifth-generation and beyond networks are positive factors in realizing the era of the Internet of Things and hyperlinked society by enabling enhanced Mobile Broadband, massive Machine-Type Communications, and ultra-Reliable Low-Latency Communications (uRLLCs) [1]-[3]. Known as one of the key services, uRLLC requires the reliability more than $99.9999 \%$, which refers to packet error rate lower than $10^{-5}$ [4], [5], and latency around $1-10 \mathrm{~ms}$ [6]. Inspired by these requirements, short-packet communications (SPCs) with finite-blocklength have become a potential solution, where some pioneering frameworks on SPCs have been considered in the literature. For example, Polyanskiy et al. in [7] explored the approximation of the maximum achievable rates as a function of error probability and blocklength. Meanwhile, Yang et al. in [8] determined the appropriate blocklength for the best ergodic capacity. Motivated by the pioneering works, the outstanding performance

N. T. Y. Linh and V. N. Q. Bao are with the Wireless Communications Department, Posts and Telecommunications Institute of Technology, Ho Chi Minh City 710372, Vietnam (email: linhnty.ncs@hcmute.edu.vn; baovnq@ptithcm.edu.vn).

N. H. Tu is with the Department of Smart Energy Systems, Seoul National University of Science and Technology, Seoul 01811, South Korea, and the Department of Computer Engineering, Ho Chi Minh City University of Transport, Ho Chi Minh City 710372, Vietnam (email: tu.ngo@ut.edu.vn).

P. N. Son is with the Department of Electrical and Electronic Engineering, Ho Chi Minh City University of Technology and Education, Ho Chi Minh City 710372, Vietnam (email: sonpndtvt@hcmute.edu.vn).

V. N. Q. Bao is the corresponding author. and capability of SPCs to support the aforementioned uRLLC requirements has been well confirmed with the integrations of various technologies and network models, such as the nonorthogonal multiple access [9], energy harvesting [6], and the multiple-input multiple-output (MIMO) systems [10], etc.

Besides that, the relaying system is known as an effective solution to extend coverage, limit transmit power support, and improve the efficiency of data transmission by using intermediate relaying nodes to support information transmission and reception over short distances [11]. Recently, many scientists have paid more attention to work on SPCs in relay networks to enhance system performance and fulfill service requirements for $5 \mathrm{G}$ and beyond networks. Specifically, Yifan $\mathrm{Gu}$ et al. in [12] compared the performance of full-duplex relaying (FDR) and half-duplex relaying (HDR) dual-hop networks under SPCs in terms of the block error rate (BLER). The results of [12] have revealed that the FDR scheme achieves better performance than that of HDR with less stringent BLER requirements and low transmit power constraints. In [13], a dual-hop hybrid automatic repeat request IoT network was investigated with SPCs, where the users utilized either selection combining or maximum ratio combining (MRC) to detect the signals. Analyzing results in [13] have confirmed that the MRC scheme achieves superior performance compared to the SC scheme with big-enough transmit power and a long-enough blocklength. Subsequently, in [14], Makki et al. considered SPCs in multirelay networks, where relays applied either amplitude-andforward (AF) or decode-and-forward (DF) schemes to transmit and process the information. It is shown that the e2e throughput of the DF scheme achieves better performance than that of the AF scheme when increasing the number of hops in a wider range of decoding delays. A joint optimization investigation of power allocation (PA) and relay location (RL) configurations for multihop MIMO relay networks with SPCs was provided in [15]. Meanwhile, the work [16] considered the partial relayselection (PRS) technique, in which the best relay was selected to forward the signal to the destination. The results in [16] have yielded a tradeoff that increasing SNR and blocklength improve system performance while increasing latency as the longer packets.

In this paper, to leverage the above aforementioned benefits of the relay networks, we first study a DF multi-relay scheme in a dual-hop network with SPCs to support not only ultrareliability but also low-latency, where the PRS technique is utilized to determine the best relay having the highest SNR among multiple relays and the destination uses the MRC technique for multiple installed antennas to achieve the highest diversity gain. Our main contributions are summarized as follows: 


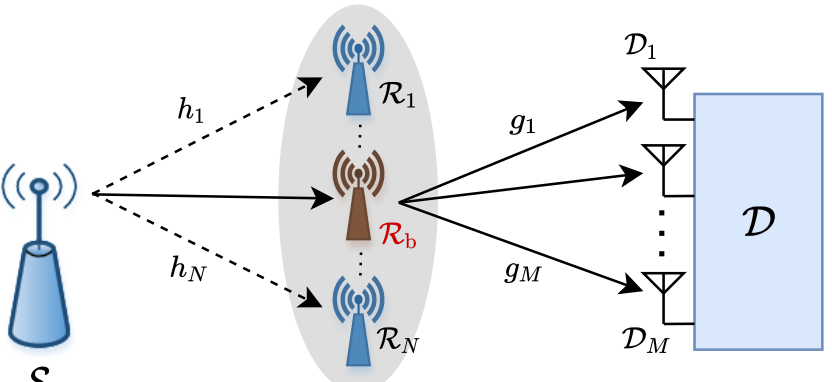

$\mathcal{S}$

Fig. 1: A dual-hop PRS/MRC relay system with SPCs.

- We derive the approximated and asymptotic closed-form expressions of the e2e BLERs for both the selective DF (SDF) and fixed DF (FDF) schemes in the finiteblocklength regime and quasi-static Rayleigh fading channels. The result indicates that the BLERs of the two schemes are identical in the average and high-SNR regions but distinguished in the low-SNR regime.

- We also investigate the performance in terms of e2e latency and system throughput to provide a comprehensive overview of not only ultra-reliability but also low-latency standpoints.

- We propose two asymptotic approaches for the e2e BLER, in which the second asymptotic approach is a way to validate the correctness of the first approach. This reveals that both of the asymptotic approaches for the e2e BLER perfectly match with each other.

- We analyze two important practical optimization problems, including PA and RL, which are obtained in the closed-form expressions for the designed configurations.

The remaining of this paper is organized as follows. In Section [I] we describe the proposed system model and derive the preliminary essential cumulative distribution function (CDF) forms. The BLER analysis of the proposed system model with both approximated and asymptotic expressions is presented in Section IIII Subsequently, the optimization problems of PA and RL are analyzed in Section IV Numerical and simulation results are discussed in Section $\mathrm{V}$ Eventually, the conclusion is provided in Section VI

\section{SYSTEM MODEL}

As illustrated in Fig. 1. we consider a dual-hop network consisting of a based station performed as a source $(\mathcal{S}), N$ single antenna relays $\mathcal{R}_{n}(n=\overline{1, N})$, and a destination $(\mathcal{D})$ installed with antennas $\mathcal{D}_{m}(m=\overline{1, M}){ }^{1}$. We assume that there is no direct link between the source and destination. The system operates in a time-division multiple-access method with two consecutive time slots via the help of the relays.

In the first time slot, the source transmits its signals to all relays. According to the PRS scheme, only the relay having the highest SNR is selected as a forwarder for the next hop.

\footnotetext{
${ }^{1}$ The value of $n=\overline{1, N}$ and $m=\overline{1, M}$ will be used throughout this paper if we do not specifically mention.
}

Denoting $\mathcal{R}_{b}$ as the selected relay in the PRS scheme among a set of $N$ relays [17], [18], we have

$$
b=\arg \max _{n=1, \ldots, N} \gamma_{\mathcal{S} \mathcal{R}_{n}},
$$

where $\gamma_{\mathcal{S} \mathcal{R}_{b}}$ denotes the output SNR in the first hop and $\gamma_{\mathcal{S} \mathcal{R}_{n}}$ is the SNR of the link from $\mathcal{S} \rightarrow \mathcal{R}_{n}$. Here, $\gamma_{\mathcal{S} \mathcal{R}_{n}}$ follows the exponential distribution with the average SNR being calculated as

$$
\bar{\gamma}_{\mathcal{S R}_{n}}=\frac{P_{\mathcal{S}}}{\sigma^{2}} \mathbb{E}\left\{\left|h_{n}\right|^{2}\right\}=\frac{P_{\mathcal{S}}}{\sigma^{2}} \Omega_{\mathcal{S R}_{n}}
$$

where $P_{\mathcal{S}}$ is the transmit power of $\mathcal{S}, h_{n}$ and $\Omega_{\mathcal{S} \mathcal{R}_{n}}$ are the channel coefficient and average channel gain of the link from $\mathcal{S} \rightarrow \mathcal{R}_{n}$, respectively, and $\sigma^{2}$ denotes the additive white Gaussian noise variance. The CDF of $\gamma_{\mathcal{S} \mathcal{R}_{b}}$ is given as

$$
F_{\gamma_{\mathcal{S} \mathcal{R}_{b}}}(\gamma)=\prod_{n=1}^{N} \operatorname{Pr}\left(\gamma_{\mathcal{S R}_{n}} \leq \gamma\right)=\prod_{n=1}^{N} F_{\gamma_{\mathcal{S R}}}(\gamma)
$$

We assume that the channel coefficients in each hop are quasi-static block-fading channels [8], where channel fading coefficients remain the constant value during each transmission block and change independently in the another transmission. The independent and identically distributed (i.i.d.) channels are also assumed with the links of $\mathcal{S} \rightarrow \mathcal{R}_{b}$ and $\mathcal{R}_{b} \rightarrow \mathcal{D}_{m}$. In such a scenario, the average SNRs for all of the branches $\bar{\gamma}_{\mathcal{S R}}$ are the same, i.e., $\bar{\gamma}_{\mathcal{S} \mathcal{R}_{n}}=\bar{\gamma}_{\mathcal{S R}}$. As all links are independently faded, the CDF of the output SNR in the first hop $\gamma_{\mathcal{S} \mathcal{R}_{b}}$ is given by

$$
\begin{aligned}
F_{\gamma_{\mathcal{S} \mathcal{R}_{\mathrm{b}}}}(\gamma) & =\left[1-\exp \left(-\frac{\gamma}{\bar{\gamma}_{\mathcal{S R}}}\right)\right]^{N} \\
& =\sum_{n=1}^{N}(-1)^{n-1}\left(\begin{array}{c}
N \\
n
\end{array}\right)\left[1-\exp \left(-\frac{n \gamma}{\bar{\gamma}_{\mathcal{S R}}}\right)\right],
\end{aligned}
$$

In the second time slot, the best relay node uses DF technique to decode successfully the received signal and transfer this signal to the destination $\mathcal{D}$. At the destination $\mathcal{D}$, the spatial diversity MRC protocol is investigated, i.e., it combines all channels $\mathcal{R}_{\mathrm{b}} \rightarrow \mathcal{D}_{m}$ at the destination. Therefore, the output SNR at the destination will increase linearly with the number of diversity branches. As a result, the SNR at the output of the MRC selector is determined by

$$
\gamma_{\mathcal{R}_{\mathrm{b}} \mathcal{D}}=\sum_{m=1}^{M} \gamma_{\mathcal{R}_{\mathrm{b}} \mathcal{D}_{m}}
$$

where $\gamma_{\mathcal{R}_{\mathrm{b}} \mathcal{D}_{m}}$ is the received SNR at the antenna $\mathcal{D}_{m}$. Under i.i.d. Rayleigh fading assumption for the link of $\mathcal{R}_{b} \rightarrow \mathcal{D}_{m}$, we also have $\bar{\gamma}_{\mathcal{R}_{\mathrm{b}} \mathcal{D}_{m}}=\bar{\gamma}_{\mathcal{R}_{\mathrm{b}} \mathcal{D}}=\frac{P_{\mathcal{R}_{\mathrm{b}}}}{\sigma^{2}} \Omega_{\mathcal{R}_{\mathrm{b}} \mathcal{D}}$, where $P_{\mathcal{R}_{\mathrm{b}}}$ is the transmit power at $\mathcal{R}_{\mathrm{b}}$ and $\Omega_{\mathcal{R}_{\mathrm{b}} \mathcal{D}}$ is the average channel gain of the link from $\mathcal{R}_{\mathrm{b}} \rightarrow \mathcal{D}$. We note that $\left|g_{m}\right|^{2}$ follows the exponential distribution so that $\gamma_{\mathcal{R}_{\mathrm{b}} \mathcal{D}_{m}}$ follows the chi-squared distribution with a mean of $M \bar{\gamma}_{\mathcal{R}_{\mathrm{b}} \mathcal{D}}$ and variance $2 M \bar{\gamma}_{\mathcal{R}_{\mathrm{b}} \mathcal{D}}$ [19]. Accordingly, the CDF of $\gamma_{\mathcal{R}_{\mathrm{b}} \mathcal{D}}$ is given by

$$
F_{\gamma_{\mathcal{R}_{\mathrm{b}} \mathcal{D}}}(\gamma)=1-\exp \left(-\frac{\gamma}{\bar{\gamma}_{\mathcal{R}_{\mathrm{b}} \mathcal{D}}}\right) \sum_{m=1}^{M} \frac{\left(\frac{\gamma}{\overline{\bar{\gamma}_{\mathcal{R}_{\mathrm{b}} \mathcal{D}}}}\right)^{m-1}}{(m-1) !} .
$$




\section{Performance AnAlysis}

In this section, we derive the approximated closed-form expression for the e2e BLER under the finite-blocklength regime. The asymptotic analysis are further studied to gain more insights into the performance behavior in the high-SNR regime. Furthermore, the performance in terms of e2e latency and throughput is also considered.

\section{A. Average BLER}

We assume that each transmission blocklength is $k$ channel uses (CUs). When $\mathcal{S}$ transmits $\beta$ information bits to $\mathcal{D}$ over $k$ during each packet transmission, the coding rate of the proposed system is $r=2 \beta / k$. For SPCs, the minimum blocklength should be larger than 100 CUs [20], the average BLER can be tightly approximated as [15. Eq. (9)]

$$
\bar{\varepsilon}_{\mathcal{X}} \approx \mathbb{E}\left\{Q\left(\frac{C\left(\gamma_{\mathcal{X}}\right)-r}{\sqrt{V\left(\gamma_{\mathcal{X}}\right) / k}}\right)\right\}
$$

where $\mathcal{X} \in\left\{\mathcal{S R}_{\mathrm{b}}, \mathcal{R}_{\mathrm{b}} \mathcal{D}\right\}, C\left(\gamma_{\mathcal{X}}\right)=\log _{2}\left(1+\gamma_{\mathcal{X}}\right)$ is the Shannon channel capacity, $V\left(\gamma_{\mathcal{X}}\right)=\left(1-\frac{1}{\left(1+\gamma_{\mathcal{X}}\right)^{2}}\right)\left(\log _{2} e\right)^{2}$ is the channel dispersion measuring the random variation of a channel compared to a defined channel for the same capacity, $\mathbb{E}\{$.$\} is the expectation operator, and Q($.$) is the Gaussian Q-$ function [21]. From (8), we observe that the form of Gaussian Q-function is too complicated, which yields that it is intractable to solve (8) directly. We now envoke the approximated linear form of $Q\left(\frac{C\left(\gamma_{\mathcal{X}}\right)-r}{\sqrt{V\left(\gamma_{\mathcal{X}}\right) / k}}\right) \approx \Phi\left(\gamma_{\mathcal{X}}\right)[20$. Eq. (14)] with

$$
\Phi\left(\gamma_{\mathcal{X}}\right)= \begin{cases}1, & \gamma_{\mathcal{X}} \leq \rho_{L}, \\ 0.5-v \sqrt{k}\left(\gamma_{\mathcal{X}}-\theta\right), & \rho_{L}<\gamma_{\mathcal{X}}<\rho_{H}, \\ 0, & \gamma_{\mathcal{X}} \geq \rho_{L},\end{cases}
$$

where $v=\frac{1}{\sqrt{2 \pi\left(2^{2 r}-1\right)}}, \theta \triangleq 2^{r}-1, \rho_{H}=\theta+\frac{1}{2 v \sqrt{k}}$, and $\rho_{L}=\theta-\frac{1}{2 v \sqrt{k}}$. Accordingly, (8) can be tightly approximated as

$$
\bar{\varepsilon}_{\mathcal{X}} \approx v \sqrt{k} \int_{\rho_{L}}^{\rho_{H}} F_{\gamma_{\mathcal{X}}}(\gamma) d \gamma
$$

By substituting (4) and (6) into (9), the average BLERs at the first and second hop are obtained, respectively, as

$$
\begin{aligned}
& \bar{\varepsilon}_{\mathcal{S R}_{\mathrm{b}}} \approx 1+v \sqrt{k} \sum_{n=1}^{N}(-1)^{n-1}\left(\begin{array}{c}
N \\
n
\end{array}\right) \frac{\bar{\gamma}_{\mathcal{S R}}}{n} \\
& \cdot\left\{\exp \left(-\frac{n \rho_{H}}{\bar{\gamma}_{\mathcal{S R}}}\right)-\exp \left(-\frac{n \rho_{L}}{\bar{\gamma}_{\mathcal{S R}}}\right)\right\} \text {, } \\
& \bar{\varepsilon}_{\mathcal{R}_{\mathrm{b}} \mathcal{D}} \approx 1-v \sqrt{k} \sum_{m=1}^{M} \frac{\bar{\gamma}_{\mathcal{R}_{\mathrm{b}} \mathcal{D}}}{(m-1) !} \\
& \text {. }\left\{\Upsilon\left(m, \frac{\rho_{H}}{\bar{\gamma}_{\mathcal{R}_{\mathrm{b}} \mathcal{D}}}\right)-\Upsilon\left(m, \frac{\rho_{L}}{\bar{\gamma}_{\mathcal{R}_{\mathrm{b}} \mathcal{D}}}\right)\right\},
\end{aligned}
$$

where $\Upsilon(\cdot, \cdot)$ is the lower incomplete Gamma function with $\Upsilon(m, x)=\int_{0}^{x} t^{m-1} \exp (-t) d t[22]$.
Proposition 1. Since the SDF scheme is considered at relays [23], where the relay node supports only when it is able to decode successfully the source information. As a result, the e2e BLER can be calculated as

$$
\bar{\varepsilon}_{\mathrm{e} 2 \mathrm{e}}^{\mathrm{SDF}}=\bar{\varepsilon}_{\mathcal{S R}_{\mathrm{b}}}+\left(1-\bar{\varepsilon}_{\mathcal{S} \mathcal{R}_{\mathrm{b}}}\right) \bar{\varepsilon}_{\mathcal{R}_{\mathrm{b}} \mathcal{D}}
$$

Otherwise, since FDF is utilized at relays [24], where the selected relay always decodes and forwards the source information without regarding the ability of prosperous decoding. In such a scenario, the e2e BLER is given by

$$
\bar{\varepsilon}_{\mathrm{e} 2 \mathrm{e}}^{\mathrm{FDF}}=\bar{\varepsilon}_{\mathcal{S} \mathcal{R}_{\mathrm{b}}}\left(1-\bar{\varepsilon}_{\mathcal{R}_{\mathrm{b}} \mathcal{D}}\right)+\left(1-\bar{\varepsilon}_{\mathcal{S} \mathcal{R}_{\mathrm{b}}}\right) \bar{\varepsilon}_{\mathcal{R}_{\mathrm{b}} \mathcal{D}} .
$$

\section{B. Asymptotic BLER}

In this subsection, to observe the qualitative conclusions on the system performance, we present the asymptotic forms of the e2e BLERs in the high-SNR regime, where the first asymptotic approach is utilized to highlight the illustration of asymptotic characteristics and the second approach is to validate the first one.

1) The first approach: In the high-SNR regime, by applying the equivalent infinitesimal series of $1-\exp (-x) \stackrel{x \rightarrow 0}{\sim} x$ to 9), the asymptotic BLER of the first hop is calculated as

$$
\tilde{\varepsilon}_{\mathcal{S R}_{\mathrm{b}}}^{(1)} \approx \frac{v \sqrt{k}\left(\rho_{H}^{N+1}-\rho_{L}^{N+1}\right)}{(N+1) \bar{\gamma}_{\mathcal{S} \mathcal{R}}^{N}} .
$$

The asymptotic BLER of the second hop is solved by envoking the helps of [22, Eq.(8352.6)] and [22, Eq.(8354.1)]. As a result, we get

$$
\tilde{\varepsilon}_{\mathcal{R}_{\mathrm{b}} \mathcal{D}}^{(1)} \approx \frac{v \sqrt{k}\left(\rho_{H}^{M+1}-\rho_{L}^{M+1}\right)}{M !(M+1) \bar{\gamma}_{\mathcal{R}_{\mathrm{b}} \mathcal{D}}^{M}} .
$$

We note the value of BLER will be very small in the highSNR range, i.e., $\bar{\varepsilon}_{\mathcal{S} \mathcal{R}_{\mathrm{b}}} \ll 1$ and $\bar{\varepsilon}_{\mathcal{R}_{\mathrm{b}} \mathcal{D}} \ll 1$. Accordingly, Proposition 1 can be simplified, yielding the e2e asymptotic BLER for the first approach as

$$
\tilde{\varepsilon}_{\mathrm{e} 2 \mathrm{e}}^{(1)} \approx \tilde{\varepsilon}_{\mathcal{S R}_{\mathrm{b}}}^{(1)}+\tilde{\varepsilon}_{\mathcal{R}_{\mathrm{b}} \mathcal{D}}^{(1)} .
$$

2) Riemann integral approximation: By revisiting (9), we find that the value of $\rho_{H}-\rho_{L}=2 \pi \sqrt{\left(2^{2 r}-1\right) / k}$ is small since $r$ is small as $k \geq 100$ [20]. In such a scenario, the first-order Riemann integral approximation $\int_{a}^{b} f(x) d x=$ $(b-a) f\left(\frac{a+b}{2}\right)$ is valid for (9). As a result, the asymptotic BLERs for the first hop and second hop are given, respectively, by

$$
\begin{gathered}
\tilde{\varepsilon}_{\mathcal{S R}_{\mathrm{b}}}^{(2)} \approx F_{\gamma_{\mathcal{S} \mathcal{R}_{\mathrm{b}}}}(\theta) \stackrel{\text { high-SNR }}{\approx}\left(\frac{\theta}{\bar{\gamma}_{\mathcal{S R}}}\right)^{N}, \\
\tilde{\varepsilon}_{\mathcal{R}_{\mathrm{b}} \mathcal{D}}^{(2)} \approx F_{\gamma_{\mathcal{R}_{\mathrm{b}} \mathcal{D}}}(\theta) \stackrel{\text { high-SNR }}{\approx} \frac{1}{M !}\left(\frac{\theta}{\bar{\gamma}_{\mathcal{R}_{\mathrm{b}} \mathcal{D}}}\right)^{M} .
\end{gathered}
$$

Similar to (16), the e2e asymptotic BLER in for the second approach is obtained as

$$
\tilde{\varepsilon}_{\mathrm{e} 2 \mathrm{e}}^{(2)} \approx \tilde{\varepsilon}_{\mathcal{S} \mathcal{R}_{\mathrm{b}}}^{(2)}+\tilde{\varepsilon}_{\mathcal{R}_{\mathrm{b}} \mathcal{D}}^{(2)} .
$$


Remark 1. We observe that the average BLER of each hop in (9) is only dependent on the CDF function of $\gamma_{\mathcal{X}}$. When $F_{\gamma_{\mathcal{X}}}(\gamma)$ becomes too complicated, the first asymptotic approach can be intractable to obtain the closed-form expression for the average BLER. Instead of using the first asymptotic method, we can utilize the Riemann integral approximation to simplify the calculation. In this paper, we do not only utilize the second context to reduce the calculating complexity but also validate the correctness of the first approach.

Remark 2 (Diversity order). By denoting the average total transmit $S N R$ as $\bar{\gamma}=P_{0} / \sigma^{2}$, the transmit power constraint with $\phi P_{\mathcal{S}}+(1-\phi) P_{\mathcal{R}_{\mathrm{b}}} \leq P_{0}$ is satified, where $\phi \in(0,1)$ denotes the PA coefficient for the source $\mathcal{S}$. The diversity order of the proposed system is determined by [25]

$$
-\lim _{\bar{\gamma} \rightarrow \infty} \frac{\log \left(\tilde{\varepsilon}_{\mathrm{e} 2 \mathrm{e}}^{(1)}\right)}{\log (\bar{\gamma})}=-\lim _{\bar{\gamma} \rightarrow \infty} \frac{\log \left(\tilde{\varepsilon}_{\mathrm{e} 2 \mathrm{e}}^{(2)}\right)}{\log (\bar{\gamma})}=N+M .
$$

\section{E2E Latency and Throughput}

The e2e latency is characterized by the average delay during transmission and decoding. Let $D(k)$ denote the delay for decoding a packet with the blocklength $k$. Based on [14, Eq. (22)], the e2e packet transmission latency (measured in the number of CUs) is given by

$$
\tau=\{k+D(k)\}\left(2-\bar{\varepsilon}_{\mathcal{S} \mathcal{R}_{\mathrm{b}}}\right),
$$

where $D(k)=\alpha k$ with $\alpha$ being a constant that represents the decoding delay factor [26, Lemma 6].

Besides that, the e2e throughput is defined as the ratio of the number of information bits successfully received at the destination and the e2e packet transmission latency, which is measured in bits per $\mathrm{CU}$ (BPCU). As a result, the e2e throughput for the considered system is expressed as

$$
\delta=\frac{\beta\left(1-\bar{\varepsilon}_{\mathrm{e} 2 \mathrm{e}}\right)}{\tau} .
$$

\section{OptimizATION IsSUES}

This section investigates the optimization problems for PA and RL to minimize the e2e BLER under the system configuration and uRLLC constraints.

\section{A. PA Optimization}

In this subsection, we will answer the important question of the relay system under the fixed coordinations of the network nodes, how is transmit power distributed to the source and relay nodes for the best system performance? The optimal PA optimization problem is given by

$$
\min _{\mathbf{P}} \bar{\varepsilon}_{\mathrm{e} 2 \mathrm{e}}(\mathbf{P}) \text { s.t. } P_{\mathcal{S}}+P_{\mathcal{R}_{\mathrm{b}}} \leq P_{0}
$$

where $\mathbf{P}$ is a set of transmit power variables, i.e., $\mathbf{P}=$ $\left(P_{\mathcal{S}}, P_{\mathcal{R}_{\mathrm{b}}}\right)$, and $P_{0}$ denotes the total transmit power budget of the system.

It is noted that the optimization problem 23 is non-convex because the objective function $\bar{\varepsilon}_{\mathrm{e} 2 \mathrm{e}}(\mathbf{P})$ is a non-convex function. Therefore, we attempt to transform 23 into a convex optimization problem. For uRLLCs, the e2e BLER must be lower than $10^{-5}$ [5], which requires a high SNR. As a result, we can invoke 19$]^{2}$ for the objective function in 23. Specifically, the problem (23) can be approximated as

$$
\min _{P_{\mathcal{S}}, P_{\mathcal{R}_{\mathrm{b}}}} \tilde{\varepsilon}_{\mathcal{S R}_{\mathrm{b}}}^{(2)}+\tilde{\varepsilon}_{\mathcal{R}_{\mathrm{b}} \mathcal{D}}^{(2)} \text { s.t. } P_{\mathcal{S}}+P_{\mathcal{R}_{\mathrm{b}}} \leq P_{0} .
$$

By inserting (17) and (18) into (24), the optimization problem (24) becomes

$\min _{P_{\mathcal{S}}, P_{\mathcal{R}_{\mathrm{b}}}}\left(\frac{\theta \sigma^{2} d_{1}^{\eta}}{P_{\mathcal{S}}}\right)^{N}+\frac{1}{M !}\left(\frac{\theta \sigma^{2} d_{2}^{\eta}}{P_{\mathcal{R}_{\mathrm{b}}}}\right)^{M}$ s.t. $P_{\mathcal{S}}+P_{\mathcal{R}_{\mathrm{b}}} \leq P_{0}$,

where $d_{1}$ is the distance between the source and multiple relays and $d_{2}$ is the distance between multiple relays and the destination. We assume the simplified pathloss model [19] with the average channel power gains $\Omega_{\mathcal{S} \mathcal{R}_{n}}=d_{1}^{-\eta}$ and $\Omega_{\mathcal{R}_{\mathrm{b}} \mathcal{D}}=d_{2}^{-\eta}$, where $\eta$ denotes the pathloss exponent.

Proposition 2. The optimization problem 25 is convex. Therefore, the Lagrangian multiplier method is beneficial to find a single global optimal solution $\mathbf{P}^{*}$.

Proof. By considering the Hessian matrix [27, Theorem. (A.2)]

$$
\mathbf{H}(\mathbf{P})=\left[\begin{array}{cc}
\frac{\partial^{2} \tilde{\varepsilon}_{\mathrm{e} 2 \mathrm{e}}^{(2)}(\mathbf{P})}{\partial P_{\mathcal{S}}^{2}} & \frac{\partial^{2} \tilde{\varepsilon}_{\mathrm{e} 2 \mathrm{e}}^{(2)}(\mathbf{P})}{\partial P_{\mathcal{S}} \partial P_{\mathcal{R}_{\mathrm{b}}}} \\
\frac{\partial^{2} \tilde{\varepsilon}_{\mathrm{e} 2}^{(2)}(\mathbf{P})}{\partial P_{\mathcal{R}_{\mathrm{b}}} \partial P_{\mathcal{S}}} & \frac{\partial^{2} \tilde{\varepsilon}_{\mathrm{e} 2 \mathrm{e}}^{(2)}(\mathbf{P})}{\partial P_{\mathcal{R}_{\mathrm{b}}}^{2}}
\end{array}\right],
$$

where

$$
\begin{gathered}
\frac{\partial^{2} \tilde{\varepsilon}_{\mathrm{e} 2 \mathrm{e}}^{(2)}(\mathbf{P})}{\partial P_{\mathcal{R}_{\mathrm{b}}} \partial P_{\mathcal{S}}}=\frac{\partial^{2} \tilde{\varepsilon}_{\mathrm{e} 2 \mathrm{e}}^{(2)}(\mathbf{P})}{\partial P_{\mathcal{S}} \partial P_{\mathcal{R}_{\mathrm{b}}}}=0, \\
\frac{\partial^{2} \tilde{\varepsilon}_{\mathrm{e} 2 \mathrm{e}}(\mathbf{P})}{\partial P_{\mathcal{S}}^{2}}=\frac{N(N+1)\left(\theta \sigma^{2} d_{1}^{\eta}\right)^{N}}{P_{\mathcal{S}}^{N+2}}>0, \\
\frac{\partial^{2} \tilde{\varepsilon}_{\mathrm{e} 2 \mathrm{e}}(\mathbf{P})}{\partial P_{\mathcal{R}_{b}}^{2}}=\frac{M(M+1)\left(\theta \sigma^{2} d_{1}^{\eta}\right)^{M}}{M ! P_{\mathcal{R}_{b}}^{M+2}}>0 .
\end{gathered}
$$

Because all diagonal elements of $\mathbf{H}(\mathbf{P})$ are positive, $\mathbf{H}(\mathbf{P})$ is positive definite. As a result, $\tilde{\varepsilon}_{\mathrm{e} 2 \mathrm{e}}^{(2)}(\mathbf{P})$ in 25 is a strictly convex function. Since the constraint is affine, the optimization problem 25 is convex, which completes the proof.

Based on Proposition 2, we use the Lagrangian multiplier method to determine $\mathbf{P}^{*}$, where the Lagrange function of 25 is given by

$$
\Delta_{1}\left(\mathbf{P}, \psi_{1}\right)=\frac{\left(\theta \sigma^{2} d_{1}^{\eta}\right)^{N}}{P_{\mathcal{S}}^{N}}+\frac{\left(\theta \sigma^{2} d_{2}^{\eta}\right)^{M}}{M ! P_{\mathcal{R}_{\mathrm{b}}}^{M}}-\psi_{1}\left(P_{\mathcal{S}}+P_{\mathcal{R}_{\mathrm{b}}}-P_{0}\right),
$$

where $\psi_{1}$ is a Lagrange multiplier constant. By considering the partial derivatives of $\Delta_{1}\left(\mathbf{P}, \psi_{1}\right)$ with respect to $P_{\mathcal{S}}, P_{\mathcal{R}_{\mathrm{b}}}$, and $P_{0}$ equal to zero, we get

$$
\begin{cases}\frac{\partial \Delta_{1}\left(\mathbf{P}, \psi_{1}\right)}{\partial P_{\mathcal{S}}}=-\frac{\left(\theta \sigma^{2} d_{1}^{\eta}\right)^{N} N}{P_{\mathcal{S}}^{N+1}}-\psi_{1}=0, & \left(\mathcal{F}_{1}\right), \\ \frac{\partial \Delta_{1}\left(\mathbf{P}, \psi_{1}\right)}{\partial P_{\mathcal{R}_{\mathrm{b}}}}=-\frac{\left(\theta \sigma^{2} d_{2}^{\eta}\right)^{M} M}{P_{\mathcal{R}_{\mathrm{b}}}^{M+1} M !}-\psi_{1}=0, & \left(\mathcal{F}_{2}\right), \\ \frac{\partial \Delta_{1}\left(\mathbf{P}, \psi_{1}\right)}{\partial \psi_{1}}=P_{\mathcal{S}}+P_{\mathcal{R}_{\mathrm{b}}}-P_{0}=0, & \left(\mathcal{F}_{3}\right) .\end{cases}
$$

${ }^{2}$ We note that since the results of the first and second asymptotic approaches are identical, the second strategy, however, provides lower calculating complexity. Therefore, we consider [19] as the objective function for simplification. 


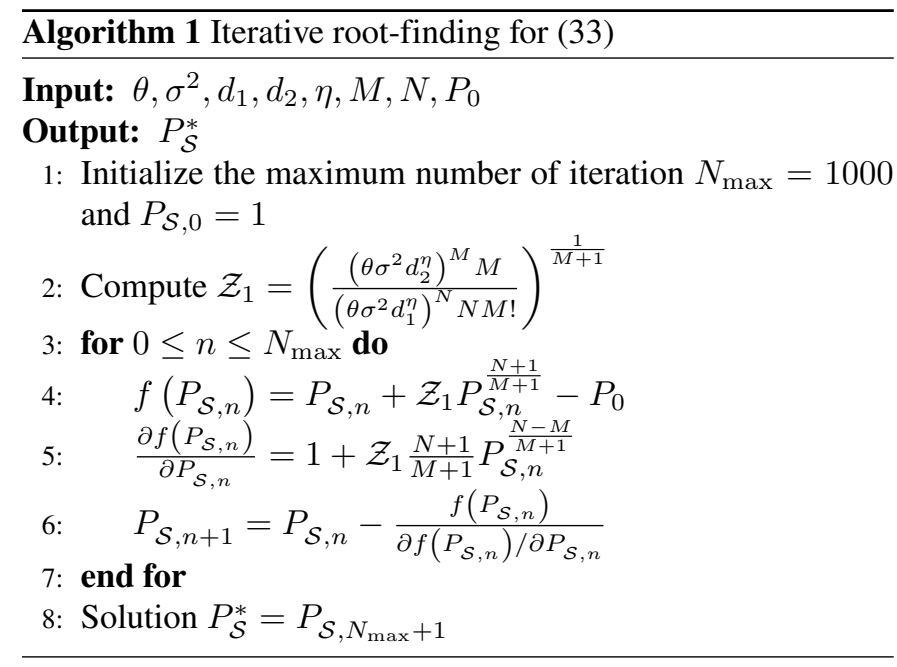

By considering $\left(\mathcal{F}_{1}\right)-\left(\mathcal{F}_{2}\right)$, we obtain

$$
P_{\mathcal{R}_{\mathrm{b}}}=\left(\frac{\left(\theta \sigma^{2} d_{2}^{\eta}\right)^{M} M}{\left(\theta \sigma^{2} d_{1}^{\eta}\right)^{N} N M !}\right)^{\frac{1}{M+1}} P_{\mathcal{S}}^{\frac{N+1}{M+1}} .
$$

By substituting 32 into $\left(\mathcal{F}_{3}\right)$, we get

$$
\underbrace{P_{\mathcal{S}}+\mathcal{Z}_{1} P_{\mathcal{S}}^{\frac{N+1}{M+1}}}_{\triangleq g\left(P_{\mathcal{S}}\right)}=P_{0}
$$

where $\mathcal{Z}_{1}=\left(\frac{\left(\theta \sigma^{2} d_{2}^{\eta}\right)^{M} M}{\left(\theta \sigma^{2} d_{1}^{\eta}\right)^{N} N M !}\right)^{\frac{1}{M+1}}$. We note that, when $P_{\mathcal{S}}$, $\mathcal{Z}_{1}>0$, and $M, N \in \mathbb{N}^{*}, g\left(P_{\mathcal{S}}\right)$ is an monotonically increasing function because of $\frac{\partial g\left(P_{\mathcal{S}}\right)}{\partial P_{\mathcal{S}}}=1+\mathcal{Z}_{1} \frac{N+1}{M+1} P_{\mathcal{S}}^{\frac{N-M}{M+1}}>0$. Furthermore, the right hand side of 33 is a constant function. When $g(0)=0, g\left(P_{\mathcal{S}}\right)>0$, and $P_{0}>0$, the equation (33) only has a unique solution. To solve (33), we consider the following proposition.

Proposition 3 (Iterative root-finding algorithm). We consider $f(x)$, from a well-chosen value $x_{0}$, the iterations for a unique root of $f(x)$ can be determined as

$$
x_{n+1}=x_{n}-\frac{f\left(x_{n}\right)}{\partial f\left(x_{n}\right) / \partial x_{n}} .
$$

Eventually, $x_{n+1}$ converges to a unique root.

Proof. Based on the definition of the derivative, we have $\frac{\partial f\left(x_{0}\right)}{\partial x_{0}}=\lim _{x \rightarrow x_{0}} \frac{f(x)-f\left(x_{0}\right)}{x-x_{0}}$. This also means

$$
\frac{\partial f\left(x_{0}\right)}{\partial x_{0}} \rightarrow \frac{f(x)-f\left(x_{0}\right)}{x-x_{0}},
$$

where the notation $\rightarrow$ represents the convergene. Assume that $x_{0}$ is a solution, we have $f\left(x_{0}\right)=0$. From 35, we get $\frac{\partial f\left(x_{0}\right)}{\partial x_{0}} \rightarrow \frac{f(x)}{x-x_{0}}$, which yields

$$
x-\frac{f(x)}{\partial f\left(x_{0}\right) / \partial x_{0}} \rightarrow x_{0},
$$

where $x_{0}$ is a converged unique solution. The proof is concluded.
By utilizing Proposition 3, Algorithm 1 is utilized to find the solution $P_{\mathcal{S}}^{*}$ for $\left(33\right.$. Based on the optimal solution $P_{\mathcal{S}}^{*}$ attained from Algorithm 1 and $\left(\mathcal{F}_{3}\right)$, the optimal $P_{\mathcal{R}_{\mathrm{b}}}^{*}$ is given by

$$
P_{\mathcal{R}_{\mathrm{b}}}^{*}=P_{0}-P_{\mathcal{S}}^{*}
$$

Remark 3. We note that, in a special case with $N=M$, the equation (33) can be easily solved and yields a unique solution as

$$
\left\{\begin{array}{l}
P_{\mathcal{S}}^{*}=\phi P_{0} \\
P_{\mathcal{R}_{\mathrm{b}}}^{*}=(1-\phi) P_{0}
\end{array}\right.
$$

where $\phi=\left(1+\left(\frac{1}{M !}\right)^{\frac{1}{M+1}}\left(\frac{d_{2}}{d_{1}}\right)^{\frac{\eta M}{M+1}}\right)^{-1}$.

\section{B. RL Optimization}

In this subsection, the optimal RL configuration is designed to minimize the e2e BLER with the constraint of the normalized transmission distance and the given PA configuration. Since the original RL optimization is non-convex, we formulate the optimal RL problem as a convex optimization by utilizing the same hypotheses as those in the PA optimization problem. Specifically,

$$
\min _{\mathbf{d}} \tilde{\varepsilon}_{\mathrm{e} 2 \mathrm{e}}^{(2)}(\mathbf{d})=\min _{d_{1}, d_{2}} \tilde{\varepsilon}_{\mathcal{S} \mathcal{R}_{\mathrm{b}}}^{(2)}+\tilde{\varepsilon}_{\mathcal{R}_{\mathrm{b}} \mathcal{D}}^{(2)} \text { s.t. } d_{1}+d_{2}=D,
$$

where $\mathbf{d}=\left(d_{1}, d_{2}\right)$ and $D$ represents the normalized transmit distance. By substituting (17) and (18) into (39), we get

$$
\min _{d_{1}, d_{2}}\left(\frac{\theta \sigma^{2} d_{1}^{\eta}}{P_{\mathcal{S}}}\right)^{N}+\frac{1}{M !}\left(\frac{\theta \sigma^{2} d_{2}^{\eta}}{P_{\mathcal{R}_{\mathrm{b}}}}\right)^{M} \text { s.t. } d_{1}+d_{2}=D .
$$

By following the same footsteps as Proposition 2, it is the fact that 40 is a convex optimization problem. Therefore, the Lagrangian multiplier method is beneficial to find the global optimal solution $\mathbf{d}^{*}$. We define the Lagrangian cost function for (40) as

$$
\Delta_{2}\left(\mathbf{d}, \psi_{2}\right)=\left(\frac{\theta \sigma^{2} d_{1}^{\eta}}{P_{\mathcal{S}}}\right)^{N}+\frac{1}{M !}\left(\frac{\theta \sigma^{2} d_{2}^{\eta}}{P_{\mathcal{R}_{\mathrm{b}}}}\right)^{M}-\psi_{2}\left(d_{1}+d_{2}-D\right),
$$

where $\psi_{2}$ is a Langrange multiplier constant. Similarly, by performing the partial derivatives of $\Delta_{2}\left(\mathbf{d}, \psi_{2}\right)$ with respect to $d_{1}, d_{2}$, and $\psi_{2}$ equal to zero, we obtain

$$
\left\{\begin{array}{lll}
\frac{\partial \Delta_{2}\left(\mathbf{d}, \psi_{2}\right)}{\partial d_{1}}=\frac{\left(\theta \sigma^{2}\right)^{N}}{P_{\mathcal{S}}^{N}} N \eta d_{1}^{N \eta-1}-\psi_{2}=0, & & \left(\mathcal{G}_{1}\right), \\
\frac{\partial \Delta_{2}\left(\mathbf{d}, \psi_{2}\right)}{\partial d_{2}}=\frac{\left(\theta \sigma^{2}\right)^{M}}{P_{\mathcal{R}_{\mathrm{b}}}^{M !}} M \eta d_{2}^{M \eta-1}-\psi_{2}=0, & & \left(\mathcal{G}_{2}\right), \\
\frac{\partial \Delta_{2}\left(\mathbf{d}, \psi_{2}\right)}{\partial \psi_{1}}=d_{1}+d_{2}-D=0, & & \left(\mathcal{G}_{3}\right) .
\end{array}\right.
$$

By considering $\left(\mathcal{G}_{1}\right)-\left(\mathcal{G}_{2}\right)$, we get

$$
d_{2}=\left(\frac{\left(\theta \sigma^{2}\right)^{N} N P_{\mathcal{R}_{\mathrm{b}}}^{M} M !}{\left(\theta \sigma^{2}\right)^{M} M P_{\mathcal{S}}^{N}}\right)^{\frac{1}{M \eta-1}} d_{1}^{\frac{N \eta-1}{M \eta-1}}
$$




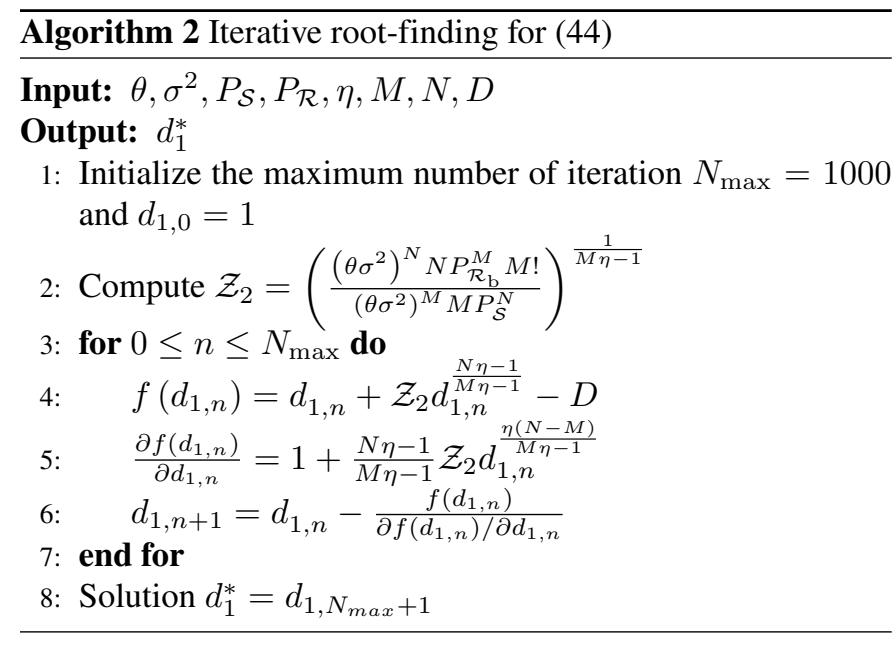

Subsequently, by inserting 43 into $\left(\mathcal{G}_{3}\right)$, we obtain

$$
d_{1}+\mathcal{Z}_{2} d_{1}^{\frac{N \eta-1}{M \eta-1}}=D
$$

where $\mathcal{Z}_{2}=\left(\frac{\left(\theta \sigma^{2}\right)^{N} N P_{\mathcal{R}_{\mathrm{b}}}^{M} M !}{\left(\theta \sigma^{2}\right)^{M} M P_{\mathcal{S}}^{N}}\right)^{\frac{1}{M \eta-1}}$. Similar to 33, 44 also has a unique solution. By utilizing Proposition 3, we design Algorithm 2 to determine the optimal solution $d_{1}^{*}$ for (44). Eventually, we replace the obtained $d_{1}^{*}$ from Algorithm 2 into $\left(\mathcal{G}_{3}\right)$, which yields

$$
d_{2}^{*}=D-d_{1}^{*}
$$

Remark 4. For a special case with $N=M$, the optimal $R L$ configuration is given by

$$
\left\{\begin{array}{l}
d_{1}^{*}=D\left(1+(M !)^{\frac{1}{M \eta-1}}\left(\frac{P_{\mathcal{R}_{\mathrm{b}}}}{P_{\mathcal{S}}}\right)^{\frac{M}{M \eta-1}}\right)^{-1}, \\
d_{2}^{*}=D-d_{1}^{*} .
\end{array}\right.
$$

\section{Simulation Results and Discussion}

In this section, we perform Monte Carlo simulations to verify our theoretical analysis presented in the previous sections.

\section{A. Performance Evaluation}

For parameter settings, we assume the information with $\beta=256$ bits, blocklength $k=256 \mathrm{CUs}$, and pathloss exponent $\eta=3$. We consider the asymmetric system with $d_{1}=0.7 \cdot D$, $d_{2}=0.3 \cdot D, P_{\mathcal{S}}=0.7 \cdot P_{0}$, and $P_{\mathcal{R}_{\mathrm{b}}}=0.3 \cdot P_{0}$. The normalized transmission distance $D=10$ is assumed.

First, we compare the e2e BLER between the FDF and SDF schemes, as shown in Fig. 2. Here, $N=3$ and $M=3$ are assumed for the parameter settings. In this figure, we observe that although the e2e BLERs of the SDF and FDF schemes are identical in the medium and high-SNR regimes, their performance, however, is different in the low-SNR range. In particular, the performance of FDF is better than that of the SDF scheme in the low-SNR regime. Consequently, we will only consider the FDF scheme for the later figures. Importantly, the simulation results completely coincide with the theoretical analysis results, which confirm the correctness of our analysis.

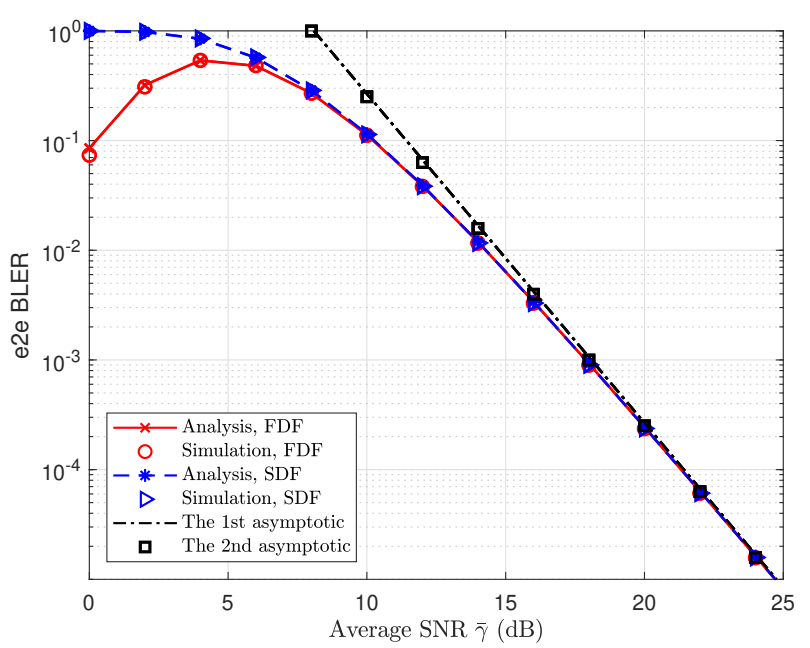

Fig. 2: The performance comparison between the FDF and SDF schemes, where $N=3$ and $M=3$.

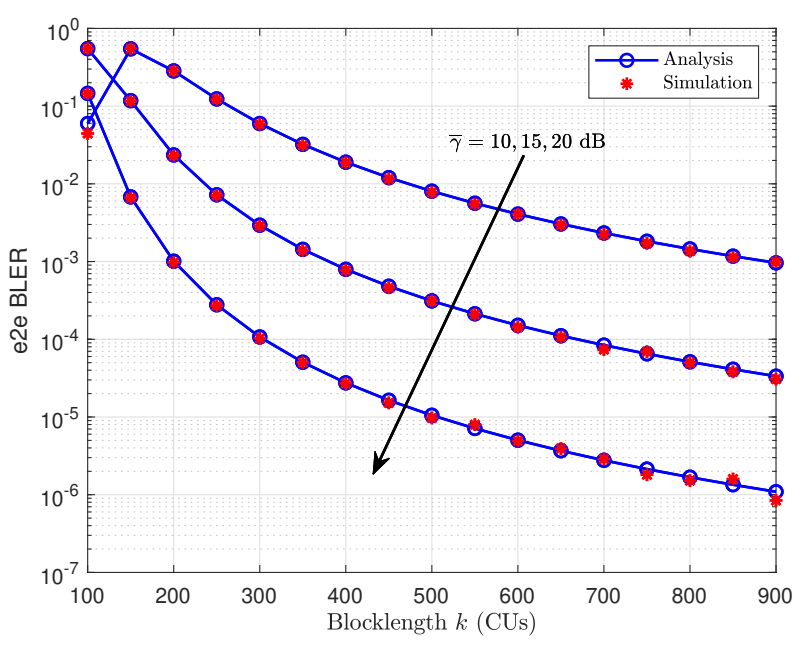

Fig. 3: The effect of blocklength $k$ on the e2e BLER, where $N=3$ and $M=3$.

Fig. 3 shows the effect of blocklength on the system performance in three typical cases of average SNRs as $10 \mathrm{~dB}, 15 \mathrm{~dB}$ and $20 \mathrm{~dB}$, where the parameters of $N=3$ and $M=3$ are set. In Fig. 3, we recognize that the system performance is improved by increasing the average SNR and blocklength. However, we note that the longer blocklength also causes the higher e2e latency, as depicted in Fig. 7. This tradeoff on the blocklength value should be conservatively considered. For a given average SNR $\bar{\gamma}=20 \mathrm{~dB}$, to ensure the reliability requirement in the order of e2e BLER of $10^{-5}$, the blocklength of 500 CUs can be chosen.

Subsequently, Fig. 4 investigates the effect of the number of relays and received antennas at the destination on the system BLER, which is presented in three-dimensional space. Each plane represents the BLER analysis result with a specific average transmit SNR, where we consider three levels of $\bar{\gamma}$ as $5 \mathrm{~dB}, 10 \mathrm{~dB}$, and $15 \mathrm{~dB}$. The simulation results, which are presented by dots, are math well with the analysis plane. As seen in Fig. 4, the e2e BLERs dramatically drop when $\bar{\gamma}, N$ relays, and $M$ antennas increase, as expected. We note that, as 


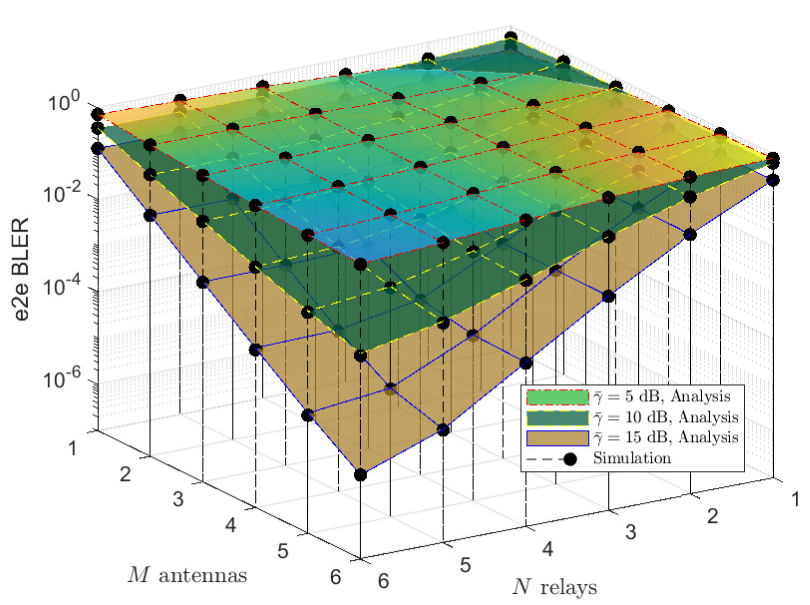

Fig. 4: The influence of the number of relays and received antennas at the destination on the system performance.

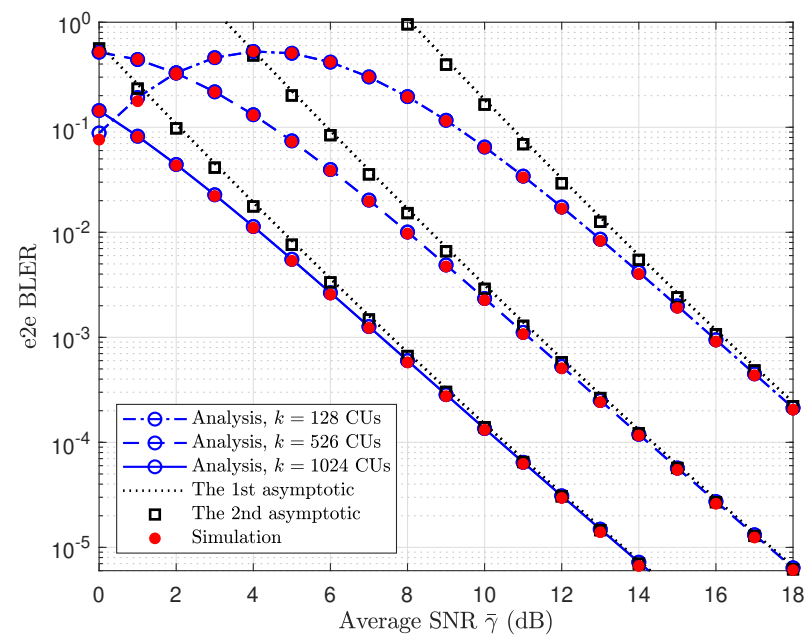

Fig. 5: The suitability between the approximated and asymptotic BLERs as a function of average SNR $\bar{\gamma}$ in dB with $N=4$ and $M=3$.

stated in Remark 2, the diversity gain of the proposed system is dependent on the design of the number of relays and received antennas at the destination, which yields the important role of the designed numbers of relays and antennas for the network.

Fig. 5 depicts the suitability between the approximated and asymptotic BLERs. Here, the results are derived for the scenario of $N=4, M=3$, and $k=128,512,1024$ CUs. As seen in Fig. 5, the e2e BLER is decreased when increasing $\bar{\gamma}$ and $k$. The theoretical analysis and simulation curves converge with the asymptotic one in the high-SNR regime. Furthermore, the second asymptotic approach is considered as a validated strategy of the first asymptotic one, where they are in a good agreement with each other.

Eventually, in Fig. 6, we can observe the system performance in terms of the e2e latency and throughput that are the crucial evaluation parameters in the uRLLC requirements, where $N=4, M=3, \bar{\gamma}=20 \mathrm{~dB}$, and $\alpha=0.5,1.5,2.5$ are assumed. While increasing blocklength can improve the

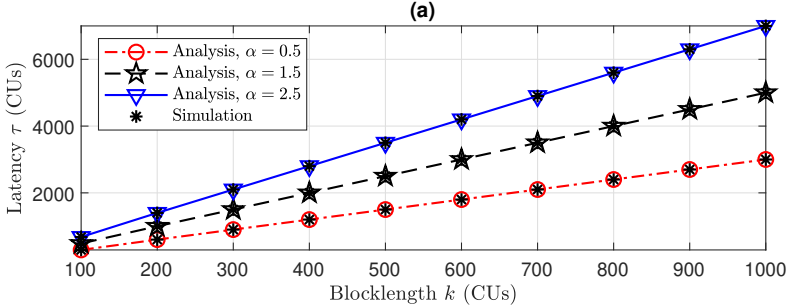

(b)

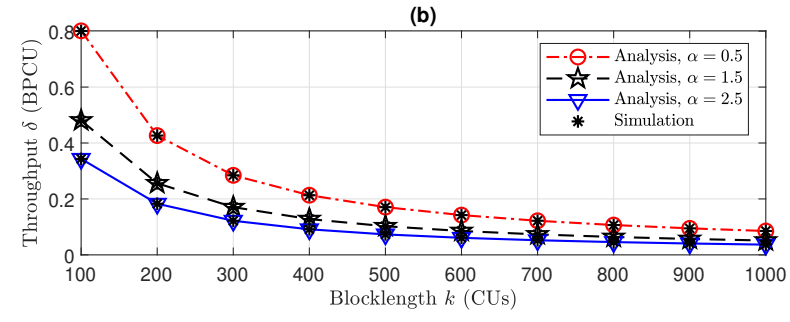

Fig. 6: The influence of the e2e latency and throughput on the blocklength, where $N=4, M=3$, and $\bar{\gamma}=20 \mathrm{~dB}$.

reliability, they suffer from the higher latency and throughput. Besides that, Fig. 6 also yields the impact of the decoding delay factor. Specifically, with the higher value of $\alpha$, the system performance is negatively affected in terms of the e2e latency and throughput. We note that, for a CU duration of $3 \mu \mathrm{s}[6]$, $\alpha=2.5$, and the parameter settings given in Fig. 6, if we choose the blocklength of $k=200$ CUs, the system will suffer from the latency of $2000 \mathrm{CUs}$ corresponding to $6 \mathrm{~ms}$, which satisfies the low-latency constraint of uRLLCs to serve factories act applications [6].

\section{B. Performance Evaluation of Optimization Strategies}

In this subsection, we compare the system performance of the optimal and non-optimal configurations for PA and RL. For the fair comparison, $\beta=512$ bits, blocklength $k=256 \mathrm{CUs}$, normalized transmit distance $D=1$, and pathloss exponent $\eta=3$ are set in this subsection. The general case for $N$ and $M$ settings is considered with $N=6$ relays and $M=7$ antennas. Furthermore, we also show the optimization performance in a special case with $N=M=4$, where the optimal configurations of PA and RL are dominated in (38) and (46).

Fig. 7 shows the performance comparison between the optimal and non-optimal PA configurations in both the general and special cases of $N$ and $M$. In this scheme, the symmetric channels with equal transmit distance are assumed, i.e., $d_{1}=d_{2}=0.5 \cdot D$. To highlight the outstanding performance of the optimal PA configuration designed in Section IV-A we consider the non-optimal scheme with the equal PA, i.e., $P_{\mathcal{S}}=P_{\mathcal{R}_{\mathrm{b}}}=0.5 \cdot P_{0}$. Fig. 7 reveals that the optimal PA performance is significantly higher than that of the nonoptimization in the entire range of the average transmit SNR for both the general and special cases. Besides, we also observe that the array gains of $0.75 \mathrm{~dB}$ and $1.75 \mathrm{~dB}$ are achieved with the $(N=M=4)$ and $(N=6, M=7)$ cases, respectively.

In the performance comparison for RL configurations, we assume that locations of the network nodes are given with $d_{1}=d_{2}=0.5 \cdot D$. In Fig. 8, the RL optimization designed in Section IV-B is compared to the performance of the non- 


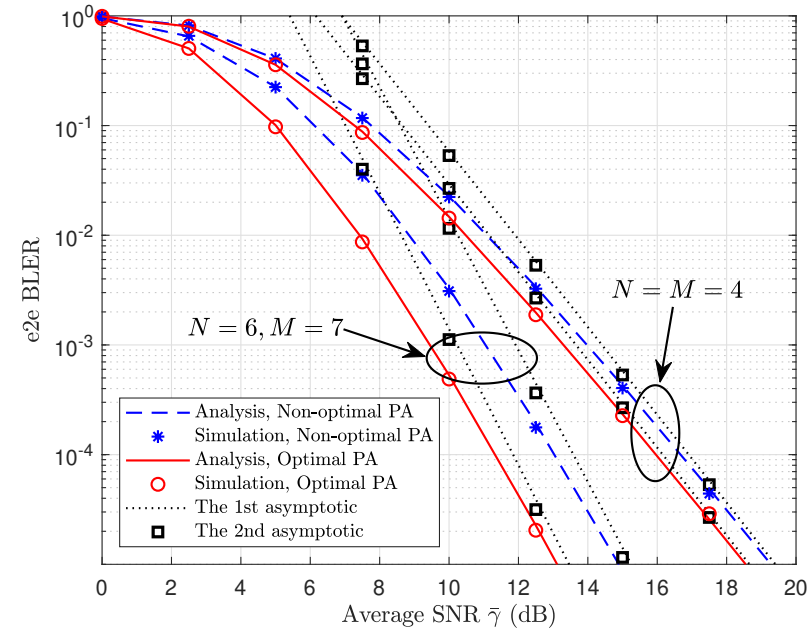

Fig. 7: The performance comparison between the optimal and non-optimal PA configurations.

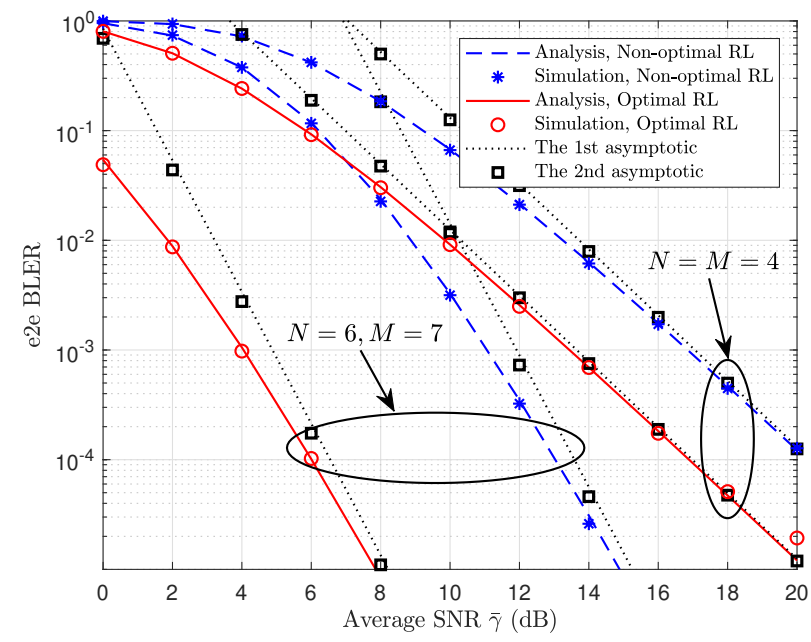

Fig. 8: The performance comparison between the optimal and non-optimal RL configurations.

optimal RL scheme with $P_{\mathcal{S}}=P_{\mathcal{R}_{\mathrm{b}}}=0.5 \cdot P_{0}$. First, we recognize that the performance behavior shown in Fig. 8 is the same as Fig. 7. which confirms the superior performance of the designed optimal RL configuration. However, the array gains achieved in Fig. 8 are much higher than that of Fig. 7 . Specifically, the array gains of $3.4 \mathrm{~dB}$ and $7 \mathrm{~dB}$ are achieved with the $(N=M=4)$ and $(N=6, M=7)$ cases, respectively.

\section{CONCLUSIONS}

In this paper, we have investigated SPCs for the dual-hop DF relaying system. The proposed relay network is beneficial in the context of limited transmit power and co-channel noise degradation, whereas the SPC framework is considered to achieve uRLLCs. In the quasi-static Rayleigh fading channels and finite-blocklength regime, the approximated e2e BLERs for both the SDF and FDF schemes are derived from the closedform expression, from which the e2e latency and throughput are also investigated to provide a performance overview of both ultra-reliability and low-latency. Subsequently, the asymptotic e2e BLER is considered to get more insights into the performance behavior in the high-SNR regime. Furthermore, two optimization strategies of PA and RL are analyzed to minimize the e2e BLER objective function under the system constraints. The results have shown that the BLERs of the SDF and FDF schemes are identical in the average and high-SNR regimes but different in the low-SNR range. Besides that, two asymptotic approaches for the e2e BLER are well matched with each other. The simulation and analysis results are also in good agreement and converge to the asymptotic curve in the highSNR regime, which validates the correctness of our analysis. Most importantly, the performance of the designed PA and RL optimization schemes is significantly better than the nonoptimal configuration.

\section{REFERENCES}

[1] M. Carugi, "Key features and requirements of 5G/IMT-2020 networks," in ITU Arab Forum on Emerg. Technol., Algiers, Algeria, Feb. 2018.

[2] J. Navarro-Ortiz, P. Romero-Diaz, S. Sendra, P. Ameigeiras, J. J. RamosMunoz, and J. M. Lopez-Soler, "A survey on 5G usage scenarios and traffic models," IEEE Commun. Surveys Tuts., vol. 22, no. 2, pp. 905929, Feb. 2020.

[3] N. N. Dao, Q. V. Pham, N. H. Tu, T. T. Thanh, V. N. Q. Bao, D. S. Lakew, and S. Cho, "Survey on aerial radio access networks: Toward a comprehensive 6G access infrastructure," IEEE Commun. Surveys Tuts., vol. 23, no. 2, pp. 1193-1225, Secondquarter 2021.

[4] K. I. Pedersen, F. Frederiksen, G. Berardinelli, and P. E. Mogensen, "The coverage-latency-capacity dilemma for TDD wide area operation and related 5G solutions," in 2016 IEEE 83rd Veh. Technol. Conf. (VTC Spring), May 2016, pp. 1-5.

[5] P. Popovski, J. J. Nielsen, C. Stefanovic, E. De Carvalho, E. Strom, K. F. Trillingsgaard, A.-S. Bana, D. M. Kim, R. Kotaba, J. Park et al., "Wireless access for ultra-reliable low-latency communication: Principles and building blocks," Ieee Network, vol. 32, no. 2, pp. 16-23, Mar.-Apr. 2018.

[6] O. L. A. López, E. M. G. Fernández, R. D. Souza, and H. Alves, "Ultrareliable cooperative short-packet communications with wireless energy transfer," IEEE Sensors J., vol. 18, no. 5, pp. 2161-2177, Apr. 2018.

[7] Y. Polyanskiy, H. V. Poor, and S. Verdú, "Channel coding rate in the finite blocklength regime," IEEE Trans. Inf. Theory, vol. 56, no. 5, pp. 23072359, May 2010.

[8] W. Yang, G. Durisi, T. Koch, and Y. Polyanskiy, "Quasi-static multipleantenna fading channels at finite blocklength," IEEE Trans. Inf. Theory, vol. 60, no. 7, pp. 4232-4265, Apr. 2014

[9] X. Sun, S. Yan, N. Yang, Z. Ding, C. Shen, and Z. Zhong, "Shortpacket downlink transmission with non-orthogonal multiple access," IEEE Trans. Wireless Commun., vol. 17, no. 7, pp. 4550-4564, 2018.

[10] J. Zeng, T. Lv, R. P. Liu, X. Su, Y. J. Guo, and N. C. Beaulieu, "Enabling ultrareliable and low-latency communications under shadow fading by massive MU-MIMO," IEEE Internet Things J., vol. 7, no. 1, pp. 234-246, Jan. 2020.

[11] T. Cover and A. E. Gamal, "Capacity theorems for the relay channel," IEEE Trans. Inf. Theory, vol. 25, no. 5, pp. 572-584, Sep. 1979.

[12] Y. Gu, H. Chen, Y. Li, and B. Vucetic, "Ultra-reliable short-packet communications: Half-duplex or full-duplex relaying?" IEEE Wireless Commun. Lett., vol. 7, no. 3, pp. 348-351, 2017.

[13] Y. Yang, Y. Song, and F. Cao, "HARQ assisted short-packet communications for cooperative networks over Nakagami- $m$ fading channels," IEEE Access, vol. 8, pp. 151 171-151 179, Aug. 2020.

[14] B. Makki and M.-S. Alouini, "End-to-end performance analysis of delaysensitive multi-relay networks," IEEE Commun. Lett., vol. 23, no. 12, pp. 2159-2163, Dec. 2019.

[15] N. H. Tu and K. Lee, "Performance analysis and optimization of multihop MIMO relay networks in short-packet communications," IEEE Trans. Wireless Commun., early access, Dec. 07, 2021, doi: 10.1109/TWC.2021.3131205

[16] V. N. Q. Bao and T. T. Thanh, "Performance analysis of partial relay selection networks with short packet communications," in 2019 6th NAFOSTED Conf. Inf. Comput. Sci. (NICS). Hanoi, Vietnam: IEEE, Dec. 2019, pp. 23-26. 
[17] I. Krikidis, J. Thompson, S. McLaughlin, and N. Goertz, "Amplify-andforward with partial relay selection," IEEE Commun. Lett., vol. 12, no. 4, pp. 235-237, 2008.

[18] V. N. Q. Bao and H. Y. Kong, "Diversity order analysis of dual-hop relaying with partial relay selection," IEICE Trans. Commun., vol. 92, no. 12, pp. 3942-3946, 2009.

[19] A. Goldsmith, Wireless Communications. Cambridge University Press, 2005.

[20] B. Makki, T. Svensson, and M. Zorzi, "Finite block-length analysis of the incremental redundancy HARQ," IEEE Wireless Commun. Lett., vol. 3, no. 5, pp. 529-532, Aug. 2014.

[21] P. C. Sofotasios and S. Freear, "Novel expressions for the marcum and one dimensional Q-functions," 2010 7th Int. Symp. Wireless Commun. Syst., pp. 736-740, 2010.

[22] I. S. Gradshteyn and I. M. Ryzhik, Table of integrals, series, and products.
Academic press, 2014.

[23] M. Khafagy, A. Ismail, M.-S. Alouini, and S. Aissa, "On the outage performance of full-duplex selective decode-and-forward relaying," IEEE Commun. Lett., vol. 17, no. 6, pp. 1180-1183, 2013.

[24] G. Farhadi and N. C. Beaulieu, "Fixed relaying versus selective relaying in multi-hop diversity transmission systems," IEEE Trans. Commun., vol. 58, no. 3, pp. 956-965, 2010 .

[25] D. Günduz, M. A. Khojastepour, A. Goldsmith, and H. V. Poor, "Multihop MIMO relay networks: diversity-multiplexing trade-off analysis," IEEE Trans. Wireless Commun., vol. 9, no. 5, pp. 1738-1747, May 2010.

[26] B. Makki, T. Svensson, G. Caire, and M. Zorzi, "Fast HARQ over finite blocklength codes: A technique for low-latency reliable communication," IEEE Trans. Wireless Commun., vol. 18, no. 1, pp. 194-209, 2018.

[27] S. S. Rao, Engineering optimization: theory and practice. John Wiley \& Sons, 2019. 